\title{
Attentional capture by irrelevant transients leads to perceptual errors in a competitive change detection task
}

\author{
Daniel Schneider ${ }^{1}{ }^{*}$, Christian Beste $^{2}$ and Edmund Wascher ${ }^{1}$ \\ 1 Leibniz Research Centre for Working Environment and Human Factors, Dortmund, Germany \\ 2 Department of Psychology, Institute of Cognitive Neuroscience, Biopsychology, Ruhr-University of Bochum, Bochum, Germany
}

\section{Edited by:}

Dietmar Heinke, University of

Birmingham, UK

\section{Reviewed by:}

Sanjay Kumar, University of Oxford,

University of Birmingham, UK

Saber Sami, University of

Birmingham, UK

\section{*Correspondence:}

Daniel Schneider, Leibniz Research

Centre for Working Environment and

Human Factors, Ardeystraße 67,

Dortmund 44139, Germany.

e-mail: schneiderd@ifado.de

\begin{abstract}
Theories on visual change detection imply that attention is a necessary but not sufficient prerequisite for aware perception. Misguidance of attention due to salient irrelevant distractors can therefore lead to severe deficits in change detection. The present study investigates the mechanisms behind such perceptual errors and their relation to error processing on higher cognitive levels. Participants had to detect a luminance change that occasionally occurred simultaneously with an irrelevant orientation change in the opposite hemi-field (conflict condition). By analyzing event-related potentials in the EEG separately in those error prone conflict trials for correct and erroneous change detection, we demonstrate that only correct change detection was associated with the allocation of attention to the relevant luminance change. Erroneous change detection was associated with an initial capture of attention toward the irrelevant orientation change in the N1 time window and a lack of subsequent target selection processes (N2pc). Errors were additionally accompanied by an increase of the fronto-central N2 and a kind of error negativity ( $\mathrm{Ne}$ or ERN), which, however, peaked prior to the response. These results suggest that a strong perceptual conflict by salient distractors can disrupt the further processing of relevant information and thus affect its aware perception. Yet, it does not impair higher cognitive processes for conflict and error detection, indicating that these processes are independent from awareness.
\end{abstract}

Keywords: attention, awareness, N2pc, error negativity

\section{INTRODUCTION}

In complex visual scenes we need to pay attention to certain inputs at the expense of others in order to achieve intended goals (Duncan, 2006). This process is prone to fail when irrelevant information interferes with the processing of relevant inputs. Salient changes in the scene may be missed when the visual stream is shortly interrupted or overlaid by irrelevant signals (i.e., change blindness; Simons and Levin, 1997; Simons, 2000; Simons and Rensink, 2005). The present study investigates the neural mechanisms behind such such errors in the processing of relevant information by means of event-related activity in the EEG (eventrelated potentials, ERPs). In contrast to previous studies that investigated the ERP correlates of awareness, not only the correlates of higher cognitive processing are addressed but also organizational processes in perceptual mechanisms that are assumed to play a major role for awareness.

Recent theories on visual perception suggest that the aware representation of a stimulus requires iterative processing of information between higher and lower order visual areas to gain a sufficient signal quality for visual awareness (Di Lollo et al., 2000; Enns and Di Lollo, 2000; Lamme and Roelfsema, 2000; Roelfsema et al., 2002). The change blindness paradigm is a common experimental approach to distorted information processing. Change blindness is described as the phenomenon that substantial changes in the visual environment remain undetected when a distracting event occurs simultaneously (Simons and Levin, 1997; O'Regan et al., 1999). It is assumed that salient distractors capture attention in a bottom-up way and lead to an insufficient representation of relevant information at initial processing stages. As a consequence, iterative processing is prone to fail or to be interrupted and change blindness occurs (Enns and Di Lollo, 2000). Thus, both the quality of initial stimulus processing and the sharpening of relevant information by iterative processing are essential for visual awareness.

A theory that describes the generation of initial perceptual representations is the biased competition account of visual selective attention (Desimone and Duncan, 1995). It is based on the fact that neurons in the visual system are activated by signals that fall into their receptive fields. Since neurons in the visual cortex are organized in a retinotopic manner, visual input activates a spatial map with activation focused around the location of the most salient signal (Desimone and Duncan, 1995; Desimone, 1998; Chelazzi et al., 2001). However, besides the salience of incoming signals, top-down biases can influence visual feature processing toward relevant information (Desimone and Duncan, 1995; Desimone, 1998; Kastner and Ungerleider, 2001). These top-down influences are therefore able to bias the competition between incoming stimuli, which emerges due to their salience in striate, extrastriate, and temporal visual areas (Chelazzi et al., 2001; Duncan, 2006). This way, information that is either not sufficiently salient (bottomup process) or not amplified by intention (top-down effect) gets suppressed and excluded from further processing (i.e., attentional 
selection; Chelazzi et al., 1998; Reynolds and Desimone, 2003). Therefore, already sensory processing should determine whether a signal becomes aware or not.

To study the neural processes that underlie these mechanisms, ERPs can be used. Wascher and Beste (2010b) studied competitive processing of information in a change detection paradigm by specifically varying the perceptual conflict induced by relevant luminance and irrelevant orientation changes (i.e., a motion transient) in two bars presented lateral to a central fixation cross. The authors showed that only when these stimuli were presented spatially separated, the detection of a relevant luminance change was deteriorated with increasing salience of the motion transient. On neurophysiological level, the $\mathrm{N} 1$ might indicate the early integration of bottom-up and top-down influences on attentional selection, as activation at this point of processing is modulated both by stimulus salience and intentional top-down settings (Johannes et al., 1995; Mangun, 1995; Wascher and Beste, 2010a). Posterior $\mathrm{N} 1$ asymmetries in the study of Wascher and Beste (2010b) indicated that salient irrelevant motion transients dominated initial attentional selection when they were presented spatially separated to the target. This process was subsequently counteracted by top-down mechanisms reallocating attention toward the relevant change. This mechanism was reflected by the $\mathrm{N} 2 \mathrm{pc}$ as a correlate of post-selective target processing (Hickey et al., 2006, 2009), or as an indicator for a top-down shift of attention toward relevant information (Eimer, 1995; Eimer and Kiss, 2008).

Yet, besides these processes other ERP components reflect some source of top-down attentional control. A candidate for this is the fronto-central $\mathrm{N} 2$, a component that is associated with conflict monitoring (Van Veen and Carter, 2002b; Yeung and Cohen, 2006; Folstein and Van Petten, 2008). Conflict in this sense can be elicited on different levels of information processing, leading to the allocation of appropriate attentional resources that are required for conflict control (van Veen and Carter, 2005). The source of the fronto-central $\mathrm{N} 2$ is located in the anterior cingulate cortex (ACC), an area linked with conflict detection and the impulse for other systems to exert top-down control (Botvinick et al., 2001; van Veen and Carter, 2002a).

Based on the paradigm used by Wascher and Beste (2010b), the present study investigates the origin of errors in the aware processing of visual information by comparing the ERPs of correct and erroneous change detection trials. Three sources of erroneous processing are possible in such a task. First, the relevant luminance change might not be adequately represented in the initial spatial map which might shift the further processing in favor of the irrelevant motion signal. This flaw should be visible in a comparison of the posterior $\mathrm{N} 1$ asymmetries of correct and erroneous change detection trials. Second, when only the ability to select relevant information out of an undistorted representation of information in the spatial map is affected, we would expect effects on later posterior asymmetries toward the relevant luminance change $(\mathrm{N} 2 \mathrm{pc}$; Wascher and Beste, 2010a,b).

Third, when stimulus processing is undistorted but higher cognitive mechanisms that are associated with the initiation of topdown attentional control are affected, errors should be indicated by a modulation of the fronto-central N2 component. Additionally, when the assignment of information to a proper response is deficient, errors should also be reflected in an error (related) negativity (Ne or ERN; Falkenstein et al., 1990; Gehring et al., 1990), as this component is associated with error detection based on a comparison process of response representations (Gehring et al., 1993; Falkenstein et al., 2000) or with the detection of response conflict (Carter et al., 1998; Van Veen and Carter, 2002b).

\section{MATERIALS AND METHODS PARTICIPANTS}

Twenty participants (18-30 years; 10 female) took part in the present experiment. As recorded in a screening questionnaire, none of the participants reported any known neurological or psychiatric diseases. They had normal or corrected to normal vision. All participants took part in return for course credits or a payment of $€ 8 / \mathrm{h}$ and provided informed written consent prior to beginning the experiment. The local ethic committee approved this study.

\section{STIMULI AND PROCEDURE}

Participants were seated in front of a 22 -inch CRT monitor (viewing distance about $120 \mathrm{~cm}, 100 \mathrm{~Hz}$ ) in a dimly lit chamber. Stimulus presentation was controlled by a VSG 2/5 graphic accelerator (Cambridge Research Systems, Rochester, UK). The display consisted of two bars that were presented lateral to a central fixation cross in two $200 \mathrm{~ms}$ lasting frames that were subsequently presented with a break of $50 \mathrm{~ms}$ (see Figure 1). The bars were either presented in vertical or horizontal orientation and their luminance was darker or brighter than the background $\left(30 \mathrm{~cd} / \mathrm{m}^{2}\right)$ with a Fechner contrast of 0.2 (i.e., 20 or $45 \mathrm{~cd} / \mathrm{m}^{2}$ ). Between the two frames the luminance (LUM) or orientation (ORI) of a single bar could change. Additionally, these luminance and orientation changes could occur together at the same bar (LO-Unilateral LOU) or at opposed bars (LO-Bilateral - LOB). These change conditions randomly appeared with the same probability. In order to

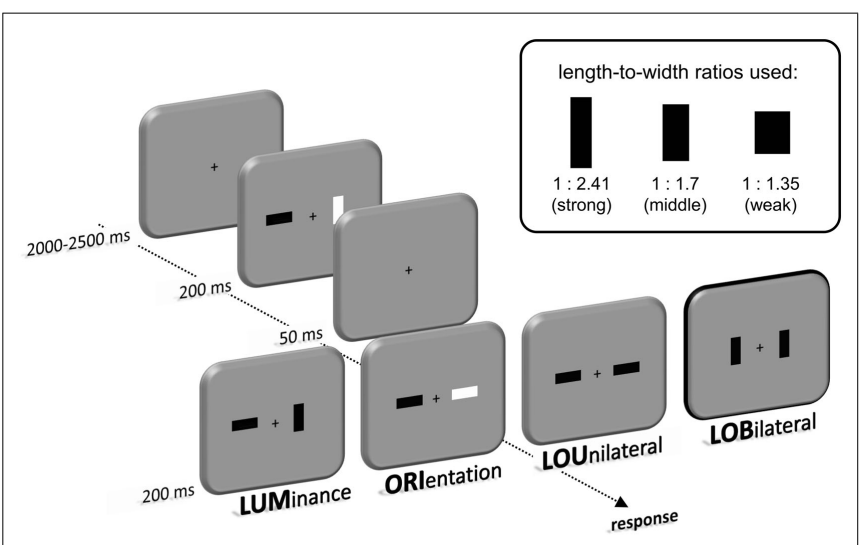

FIGURE 1 | Set-up and procedure. With a break of $50 \mathrm{~ms}$ two $200 \mathrm{~ms}$ lasting frames were presented that contained two rectangular bars lateral to a central fixation cross. Across these frames the luminance (LUM) or orientation (ORI) of these bars could change. These changes could also occur together at the same location (LOU) or spatially separated (LOB). Three different length-to-width ratios of the bars were used in order to modulate the salience of the motion transient. Participants had to respond by pressing a key at the side of the luminance change. 
vary the relative salience of relevant and irrelevant transients the bars were presented with three different length-to-width ratios for all change conditions (1:2.41, 1:1.7, and 1:1.35). The area covered by each bar remained constant at $0.76 \mathrm{~cm}^{2}$. All combinations of luminances, orientations, and length-to-width ratios were equally frequent and randomly intermixed.

The experimental procedure consisted out of three blocks with 384 trials each. The participants were instructed to respond by pressing a button with their index finger of the left or the right hand at the side of the relevant luminance change. All participants were instructed to respond as fast and accurate as possible. All trials exclusively containing an irrelevant change (ORI condition) were No-Go trials. The inter-trial-interval varied between 2000 and $2500 \mathrm{~ms}$.

\section{DATA ANALYSIS}

\section{Behavioral data}

In order to exclude trials containing premature responses, button presses were recorded from the onset of the first frame until $1500 \mathrm{~ms}$ after the change (2nd frame). Responses prior to $150 \mathrm{~ms}$ after the second frame were categorized as fast guesses, responses beyond $1000 \mathrm{~ms}$ as "too slow." These trials were excluded from further analyses. Error categories were missed responses (no response for up to $1500 \mathrm{~ms}$ ) and response errors (a button press on the wrong side or false alarms in the ORI condition between 150 and $1000 \mathrm{~ms})$.

For analyzing the overall response accuracy, an ANOVA was calculated with the repeated-measures factors "change" (LUM, ORI, LOU, LOB) and "salience" (strong, middle, and weak salience of the orientation change). In the LUM condition, the factor salience refers to the different length-to-width ratios of the presented bars. Additionally, this ANOVA was calculated separately for missed responses and response errors. In the No-Go ORI change condition, left and right button presses were defined as response errors. For missed responses and likewise for response times (RT), only the change conditions containing a luminance change were entered into analysis. Thus the factor change only included the LUM, LOU, and LOB condition. Post hoc tests (Bonferroni corrected) were applied if necessary.

\section{EEG data}

Electroencephalography (EEG) was recorded (common average served as on-line reference) from $60 \mathrm{Ag} / \mathrm{AgCl}$ active electrodes affixed across the entire scalp according to the extended 10/20 System (Pivik et al., 1993). For all participants, electrode impedance was kept below $10 \mathrm{k} \Omega$. EEG data were analyzed using Brain Vision Analyzer (v2.0.1). Eye movements were scanned continuously from two electrode pairs affixed above and below the left eye (vertical EOG) and at the outer canthi of each eye (horizontal EOG). EEG and EOG were sampled on-line with a frequency of $1 \mathrm{kHz}$ by a Quickamp DC-amplifier with a low-pass filter of $200 \mathrm{~Hz}$. No high pass filter setting was applied during EEG recording. For the data analysis, a high pass filter of $1 \mathrm{~Hz}$ and a low-pass filter of $8 \mathrm{~Hz}$ were applied. Data were re-referenced off-line to averaged mastoids. Two of the 60 channels were used for the mastoid signal that was recorded from a single electrode affixed over each mastoid. Segments with a length of $1450 \mathrm{~ms}$ (200 ms before the onset of the first frame to $1000 \mathrm{~ms}$ after stimulus change) were defined for further processing. Baseline was set to $200 \mathrm{~ms}$ preceding the first frame. The selected segments were checked off-line for artifacts (zero-lines, fast shifts, or drifts). Trials with horizontal eye movements (saccades) preceding the latency of the components of interest were excluded by calculating the difference of activation at both hEOG electrodes (left hEOG - right hEOG) and excluding trials with a fast shift in this difference wave of more than $25 \mu \mathrm{V}$ in an interval length of $40 \mathrm{~ms}$. Only trials with fast shifts in a time window of $400 \mathrm{~ms}$ around the event of interest (the change) were excluded. Based on these criteria, $85.05 \%$ of all trials in the LUM condition, $83.25 \%$ in the ORI condition, $84.27 \%$ in the LOU condition, and $80.69 \%$ in the LOB condition were used for the final analyses. The influence of remaining eye movements upon electrocortical activity was corrected by the algorithm proposed by Gratton et al. (1983).

Posterior ERPs (PO7/PO8) were used in order to compare the visuo-spatial processing between correct and erroneous change detection. Only the LOB trials served for this analysis as in this change condition enough errors were committed for showing a reliable ERP for erroneous change detection. Because both response errors and missed responses indicate that an error in the processing of relevant information occurred, these error conditions were jointly added to the average. All trials containing a correct response between 150 and $1000 \mathrm{~ms}$ were analyzed for the correct change detection condition. As no distinct peaks in the N1 time window could be measured for both correct and erroneous change detection, mean amplitudes in a time window between 150 and $250 \mathrm{~ms}$ were used. Separate ANOVAs for both conditions included the within-subjects factors "electrode" (PO7 vs. PO8; note that this factor reflects the activation over posterior electrode sites independently from change location), "asymmetry" (contra vs. ipsilateral activation), and "salience" (strong, middle, weak). The same ANOVAs were used for the N2 time window with mean amplitudes between 300 and $400 \mathrm{~ms}$.

Concerning the fronto-central N2 a maximal effect of conflict was observed for electrode FCz at about $350 \mathrm{~ms}$ for the LUM, LOU, and LOB change conditions. The ORI condition was not included in this analysis, because here a strong NO-GO P3 overlapped with the fronto-central N2. As no distinct peaks could be measured, mean amplitudes between 330 and 390 ms were entered into an ANOVA with the within-subjects factor "condition" (LUM, LOU, LOB-correct, LOB-error). While only correct trials were analyzed in the LUM and LOU conditions, erroneous, and correct change detection conditions were analyzed separately in the LOB condition. Differences in the fronto-central N2 activation between correct and erroneous change detection may be confounded by the activity related to correct and incorrect responses respectively (Van Veen and Carter, 2002b). To exclude this effect, activation in the N2 time window was additionally analyzed separately for response errors and missed responses in the LOB condition.

For the $\mathrm{Ne}$ analyses, response-locked ERPs were computed beginning $850 \mathrm{~ms}$ before and ending $500 \mathrm{~ms}$ after a response. These analyses were reduced to the LOB condition, because only in this condition behavioral results indicated that enough response errors were made for obtaining a sufficient signal-to-noise ratio in the ERP. Data were referenced off-line to averaged mastoids. 
Eye movements and all remaining artifacts were treated as in the stimulus-locked analyses. Baseline was set from 200 to $0 \mathrm{~ms}$ preceding the first stimulus. The $\mathrm{Ne}$ was identified as the maximum negative peak at electrode $\mathrm{FCz}$ in an interval beginning $100 \mathrm{~ms}$ before and ending $150 \mathrm{~ms}$ after an incorrect response. This interval was used, because participants showed a fronto-central negativity that peaked already shortly before the response. The same peak detection was run for correct trials to compare the Ne effect to the negativity related to correct responses (correct negativity or Nc; Falkenstein et al., 2000; Vidal et al., 2000). The amplitude of the $\mathrm{Ne}$ and of the Nc was measured relative to the peak of the positivity preceding both components (Gehring and Knight, 2000).

Factors with more than one degree of freedom in the numerator were Greenhouse-Geisser corrected (Vasey and Thayer, 1987). In that case, $\varepsilon$ values and $p$ values derived from the corrected degrees of freedom are reported.

\section{RESULTS}

\section{BEHAVIORAL DATA}

In the overall analysis of response accuracy, error rates varied with change condition, $F(3,57)=90.062, \varepsilon=0.524$, $p<0.001$. Pairwise comparisons (Bonferroni corrected) revealed higher error rates in the $\mathrm{LOB}$ condition compared to the remaining change conditions (all $p$ values $<0.001$ ). Additionally, error rates were increased with higher salience of the irrelevant orientation change, $F(2,38)=21.002, \varepsilon=0.986, p<0.001$. Yet, the significant salience $*$ change interaction revealed that the salience effect was restricted to the LOB condition, $F(6,114)=19.186, \varepsilon=0.559, p<0.001$. Separate analyses for misses and response errors showed that about $40 \%$ of all errors were missed responses. The rate of misses varied with change condition, $F(2,38)=29.712, \varepsilon=0.69, p<0.001$, and pairwise comparisons showed a higher number of misses in the LOB condition compared to the LUM and LOU conditions (all $p$ values $<0.001$ ). Only in the LOB condition, the rate of misses increased with the salience of the motion transient, $F(4$, $76)=7.643, \varepsilon=0.776, p<0.001$. For response errors the result pattern was identical. Error rates varied with change condition, $F(3,57)=65.771, \varepsilon=0.531, p<0.001$. This effect was due to an increase in error rates in the LOB condition compared to the remaining change conditions (all $p$ values $<0.001$ ). A salience effect was restricted to the LOB condition, $F(6,114)=11.896$, $\varepsilon=0.423, p<0.001$ Error rates separated in misses and response errors are depicted in Figure 2.

The RT analyses showed analogous results (see Figure 3). RTs varied with change condition, $F(2,38)=104.062, \varepsilon=0.717$, $p<0.001$. This effect resulted from an increase in error rates in the LOB condition compared to the LUM and LOU conditions (all $p$ values $<0.001$ ). Furthermore, only in the LOB change condition participants showed higher RTs with increasing salience of the irrelevant orientation change, $F(4,76)=35.121, \varepsilon=0.772$, $p<0.001$. In sum, error rates as well as RTs increased with higher salience of irrelevant information only when both changes appeared at different locations.

\section{EEG DATA}

Posterior ERPs for correct change detection (PO7/PO8; see Figure 4B) in the LOB condition revealed an effect of salience of the orientation change on asymmetries in the N1 time window, $F(2,38)=6.201, \varepsilon=0.733, p<0.05$. Subsequent analyses showed that only for weak orientation changes there was a posterior N1 asymmetry with higher activation contralateral to the relevant luminance transient compared to activation contralateral to the motion distractor, $F(1,19)=9.239, p<0.01$, while no asymmetry was observed for middle, $F(1,19)=0.006, p=0.941$, and strong salience levels, $F(1,19)=0.393, p=0.598$. No salience effect on posterior asymmetries in the $\mathrm{N} 1$ time range was

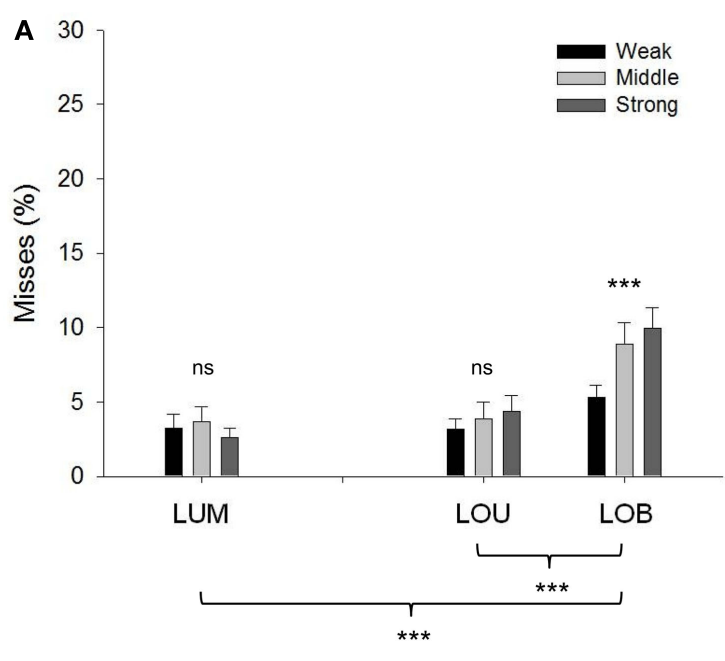

FIGURE 2 | Error rates are presented separately for misses (A) and response errors $(B)$ with error bars depicting the standard error. For both misses and response errors highest rates were shown in the LOB condition. Only in this change condition the rate of misses and response

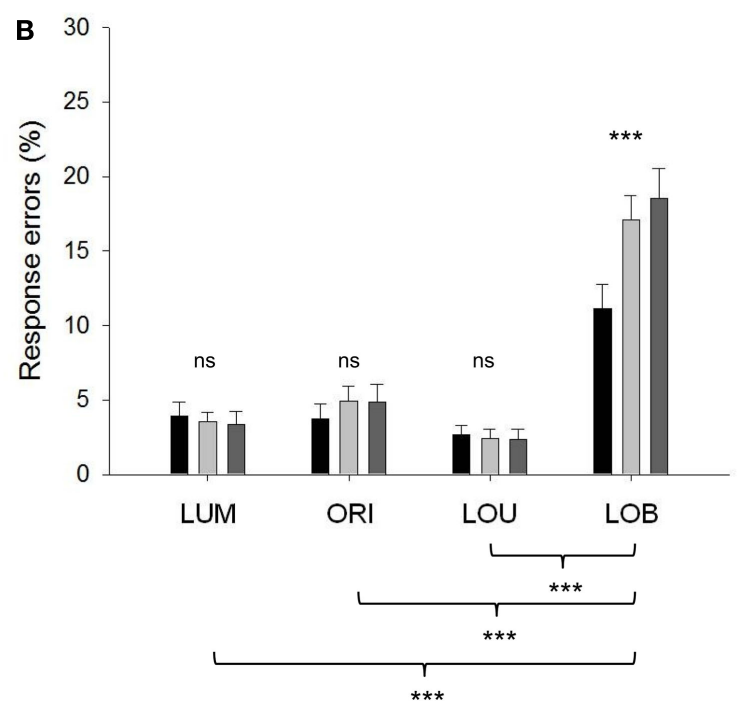

errors was enhanced with increasing salience of the irrelevant motion transient. Significant differences between change conditions (below the figure) and significant salient effects are marked. ${ }^{*} p<0.05,{ }^{*} p<0.01$, ${ }^{* * *} p<0.001$ (ns = non-significant). 


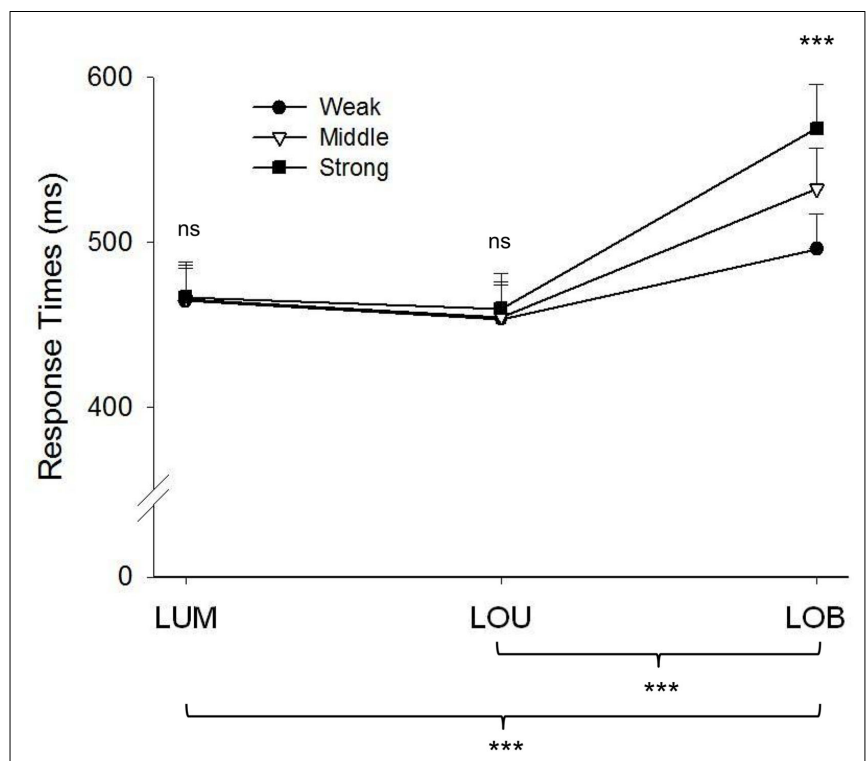

FIGURE 3 | Mean response times (RT) with error bars depicting the standard error. Compared to the remaining change conditions higher RTs were observed in the LOB condition. While no salience effect was observed for the LUM and LOU change condition RTs in the LOB condition increased with heightening salience of the motion transient. Significant differences between change conditions (below the figure) and significant salient effects are marked. ${ }^{*} p<0.05,{ }^{*} p<0.01,{ }^{* *} p<0.001$ (ns $=$ non-significant).

revealed for erroneous change detection (see Figure 4B), $F(2$, $38)=1.307, \varepsilon=0.701, p=0.277$. Yet, an asymmetry main effect with higher activation contralateral to the irrelevant orientation change appeared (see Figure 4A), $F(1,19)=5.246, p<0.05$.

Posterior asymmetries in the N2 time window were shown for correct change detection (see Figure 4B). Here, a main effect of the repeated-measures factor asymmetry with higher activation contralateral to the relevant luminance change appeared, $F(1$, $19)=38.179, p<0.001$. Additionally, this asymmetry was modulated by orientation change salience, $F(2,38)=9.005, \varepsilon=0.953$, $p<0.001$. Only for strong, $F(1,19)=37.833, p<0.001$, and for middle orientation changes, $F(1,19)=28.038, p<0.001$, an asymmetry toward the luminance change was shown. This asymmetry effect was not statistically significant for weak orientation change salience, $F(1,19)=3.597, p=0.073$. Further analyses revealed that this salience-modulated asymmetry appeared as a result of heightened activation contralateral to the luminance change with increasing salience, $F(2,38)=17.048, \varepsilon=0.941$, $p<0.001$, while no effect appeared contralateral to the motion transient, $F(2,38)=3.02, \varepsilon=0.945, p=0.064$. No posterior asymmetry in the N2 time window was shown for erroneous change detection, $F(1,19)=1.799, p=0.196$ (see Figure 4A). Additionally, no salience modulation on posterior asymmetries was evident, $F(2,38)=1.307, \varepsilon=0.993, p=0.282$. In both the N1 and $\mathrm{N} 2$ time window, no significant main effects or interactions were observed for the factor electrode (all $p$ values $<0.05$ ). Figure 5 shows posterior topographies for these asymmetric activations in the $\mathrm{N} 1$ and $\mathrm{N} 2$ time window. Higher activation contralateral to the relevant luminance change compared to activation contralateral to the motion transient is plotted on the left hemisphere, while an asymmetry toward the motion transient is plotted on the right hemisphere. Though the slight asymmetry shown in the N2pc topography for erroneous change detection was not significant on the statistical level (see prior analyses), these topographies largely resemble the effects found in the PO7/PO8 ERPs (cf. Figure 4).

Analyses concerning the fronto-central N2 (see Figure 6A) revealed a clear effect of change condition, $F(3,57)=30.659$, $\varepsilon=0.766, p<0.001$. According to pairwise comparisons, the $\mathrm{N} 2$ activation for erroneous change detection $(M=-0.965 \mu \mathrm{V}$, $\mathrm{SD}=2.203)$ was significantly higher than for correct change detection in the LOB condition $(M=-0.015 \mu \mathrm{V}, \mathrm{SD}=2.044)$. Additionally, higher activation was observed in the LOB change condition (correct change detection) compared to the LOU condition $(M=0.894 \mu \mathrm{V}, \mathrm{SD}=2.262)$, while this activation was in turn significantly higher than for single luminance changes (LUM; $M=1.411 \mu \mathrm{V}, \mathrm{SD}=2.463)$. The fronto-central N2 activation for LOB changes did not significantly differ between response errors and missed responses, $t(18)=-0.139, p=0.891$. Yet, for both response errors, $t(19)=-2.716, p<0.05$, and missed responses, $t(18)=-2.128, p<0.05$, there was a higher activation when compared to correct change detection in the LOB condition. In Figure 6B, the scalp distribution difference in the $\mathrm{N} 2$ time window is shown for erroneous and correct change detection in the LOB condition and for the LOB and LUM condition. In this time window also used for the statistical analyses (330-390 ms), a stronger negativity at fronto-central electrodes (slightly shifted to the right hemisphere) for erroneous compared to correct change detection in the LOB condition was evident. A comparable scalp distribution was shown for the LOB-LUM difference (see Figure 6B).

Concerning the $\mathrm{Ne}$ (see Figure 7A), a higher negativity was observed for erroneous compared to correct responses (Ne: $-4.27 \mu \mathrm{V}, \mathrm{SE}=0.56$; Nc: $-2.48 \mu \mathrm{V}, \mathrm{SE}=0.45), t(19)=-2.693$, $p<0.05$. Yet, for both correct and erroneous trials this negativity already peaked prior to the response. No significant difference in latency between these conditions was observed (Ne: $-14.75 \mathrm{~ms}$, $\mathrm{SE}=8.35$; Nc: $-30.7 \mathrm{~ms}, \mathrm{SE}=4.86), t(19)=1.958, p=0.065$. Figure 7B shows a fronto-central Ne topography.

\section{DISCUSSION}

The present study investigated the processes underlying errors in a change detection task using ERPs. Participants had to respond to a change of luminance that was either presented alone or simultaneously with a spatially overlapping or separated motion transient modulated in three salience levels. Behavioral results show that, compared to the other change conditions including a luminance change (LUM, LOU), the irrelevant orientation change led to deteriorated luminance change detection in the spatial conflict condition (LOB), based on error rates as well as on RT. Additionally, error rates (see Figure 2) as well as RT (see Figure 3) in the LOB change condition were heightened with increasing salience of the motion transient. Thus dependent on its salience, the motion transient impaired the processing of the relevant change when it was presented spatially separated to the target (Wascher and Beste, 2010a,b; Beste et al., 2011). In about $40 \%$ of all erroneous LOB trials this led to a miss of the relevant luminance change, indicating that participants were unaware of its presentation (see 

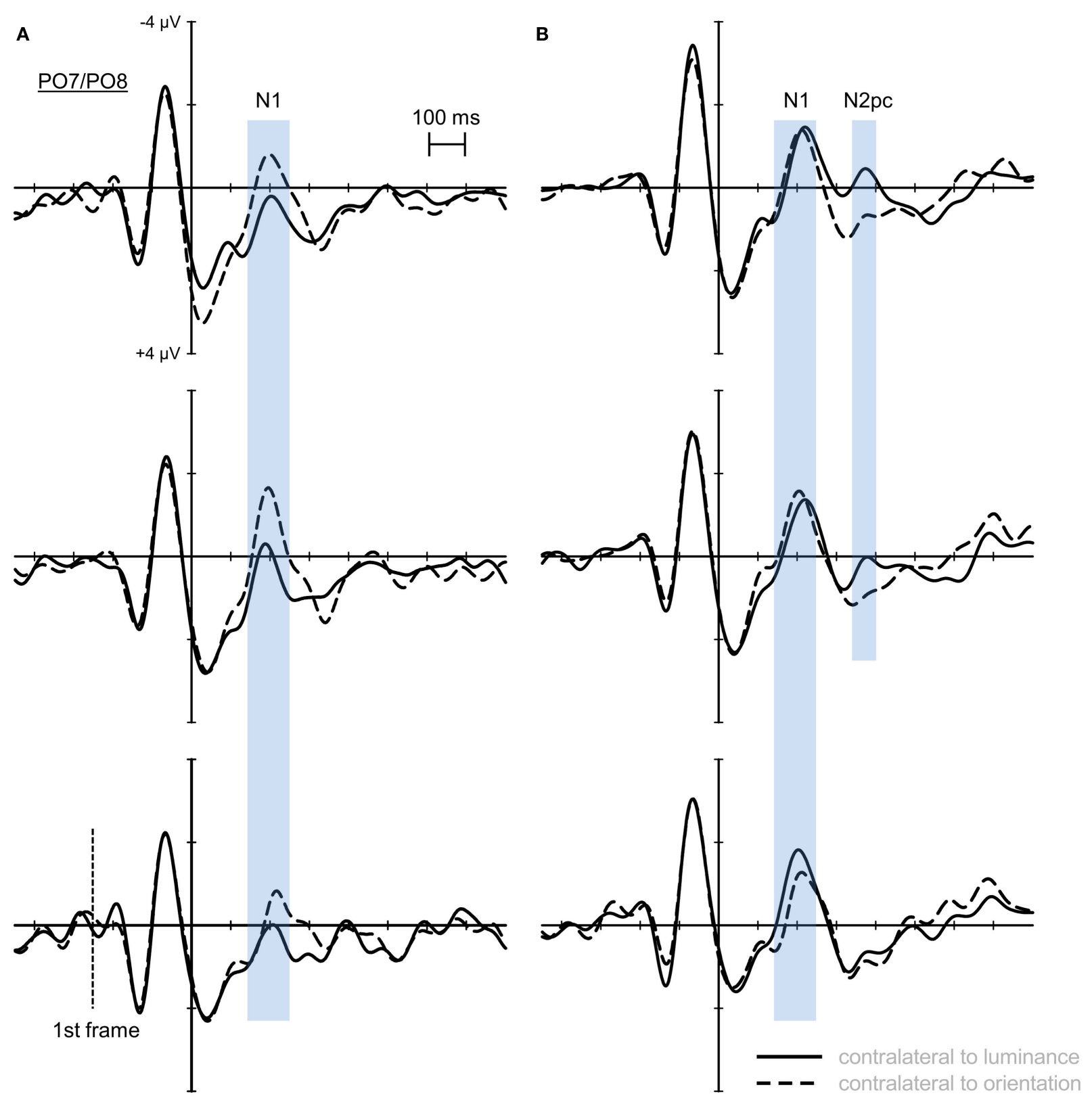

2nd frame (change)

FIGURE 4 | Posterior event-related potentials (PO7/PO8) referred to averaged mastoids for erroneous $(A)$ and correct change detection in the LOB condition (B). All figures are presented on the same scale. For erroneous change detection there was an N1 asymmetry with higher activation contralateral to the irrelevant motion for all salience levels. For correct change detection this asymmetry was modulated by the salience of the motion transient. While no asymmetry was observed when strong and middle orientation changes were presented, an asymmetry with higher activation contralateral to the relevant luminance change was present when the orientation change was weak. In the N2 time window no asymmetry was observed for erroneous change detection. For correct change detection an N2pc was shown only when there was no asymmetry toward the luminance change already in the N1 time window.
Figure 2A). The remaining errors were due to an inability in locating the relevant change that might also be a sign for incomplete target processing (see Figure 2B). The behavioral effects were comparable for these error types, indicating that they might have a common basis. Yet, given the actual experimental setting, no definite statement about a distinction or commensurability of the processes underlying these different error types can be given on the basis of behavioral results.

According to recent theories, iterative processing of information between visual areas serves to improve the representation of relevant inputs in the visual system to a level that is sufficient for awareness (Di Lollo et al., 2000; Enns and Di Lollo, 

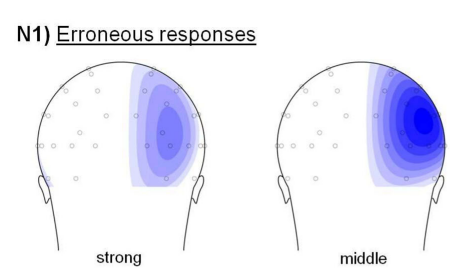

middle

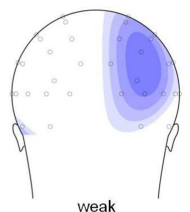

weak
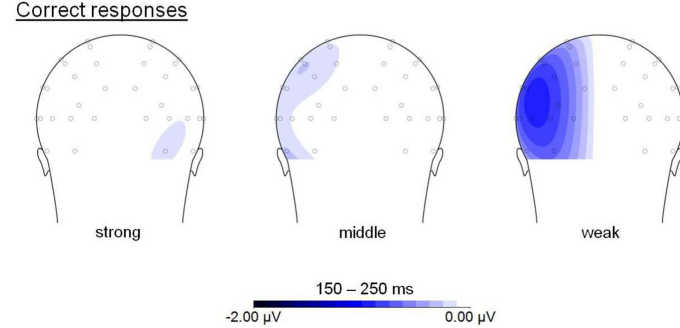

FIGURE 5 | Posterior asymmetries in the N1 and N2 time window for the LOB condition separately for erroneous (upper row) and correct change detection (lower row). Higher activation contralateral to the relevant luminance change compared to activation contralateral to the motion transient
N2pc) Erroneous responses
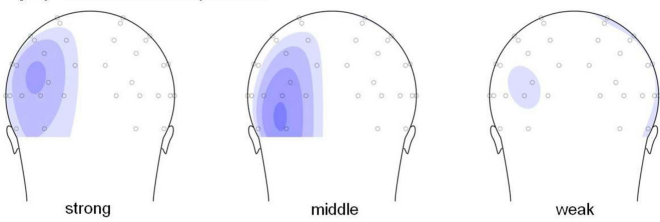

Correct responses
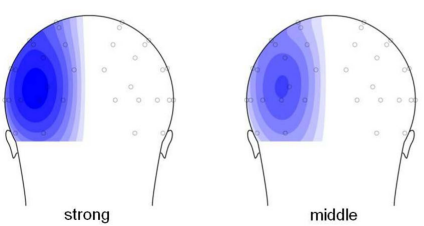

middle

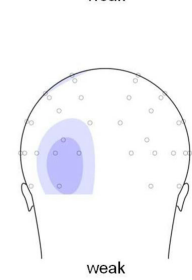

$-2.00 \mu \mathrm{V} 300-400 \mathrm{~ms}$

is plotted on the left hemisphere, while an asymmetry toward the motion transient is plotted on the right hemisphere. Topographies show scalp distributions in the time windows used for statistical analyses. All figures are presented on the same scale.

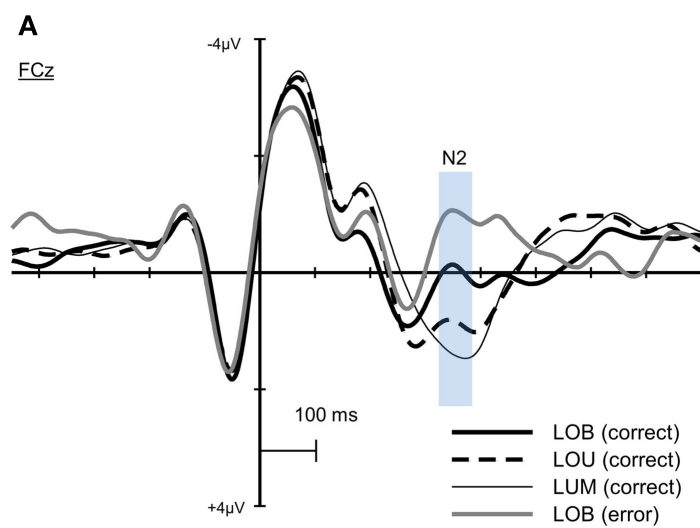

FIGURE 6 | Event-related potentials at FCz referred to averaged mastoids for the LUM, LOU, and LOB change conditions (A). For the LOB change condition, activation is displayed separately for correct and erroneous change detection. In the N2 time window the activation was modulated by the conflict induced in the respective change conditions with highest activation in the LOB change condition. Here, the fronto-central N2 activation was higher
B

LOB: Error - correct $\quad$ LOB (correct) - LUM
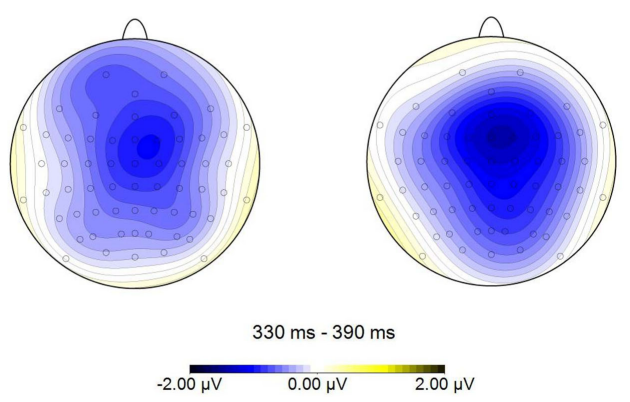

for erroneous compared to correct change detection. (B) shows the scalp distribution of the difference waves for correct and erroneous change detection in the LOB condition (error - correct) and for the LOB and LUM change condition (LOB - LUM) in the N2 time window. Both the Error-Correct and the LOB-LUM different waves indicated a fronto-central scalp distribution in the $\mathrm{N} 2$ time window.
2000; Lamme and Roelfsema, 2000; Roelfsema et al., 2002). Yet, if salient distractors affect the initial sensory representation of relevant information (Desimone and Duncan, 1995; Desimone, 1998), these processes can be eliminated and visual information, though clearly registered on the retina, cannot be consciously experienced (Di Lollo et al., 2000; Enns and Di Lollo, 2000; Crick and Koch, 2003). The present electrophysiological results of the LOB change condition show that erroneous change detection was associated with a weaker sensory representation of the relevant luminance change compared to the contralateral motion distractor. This was indicated by posterior activation in the $\mathrm{N} 1$ range that was increased contralateral to the motion transient compared to the activation contralateral to the relevant luminance change (see Figures $4 \mathrm{~A}$ and 5).

Compared to this activation pattern in the N1 time window, a higher sensory representation of the luminance change was shown when participants succeeded in detecting it (see Figure 4B). In this case, the salience of the irrelevant motion transient further modulated activation in the $\mathrm{N} 1$ range. When a weak distractor was presented, an asymmetry with higher activation contralateral to the relevant luminance change compared to ipsilateral activation appeared, while no asymmetry was evident for strong and middle salience of the motion transient (see Figures 4B and 5). This data pattern suggests that a further increase in target representation 


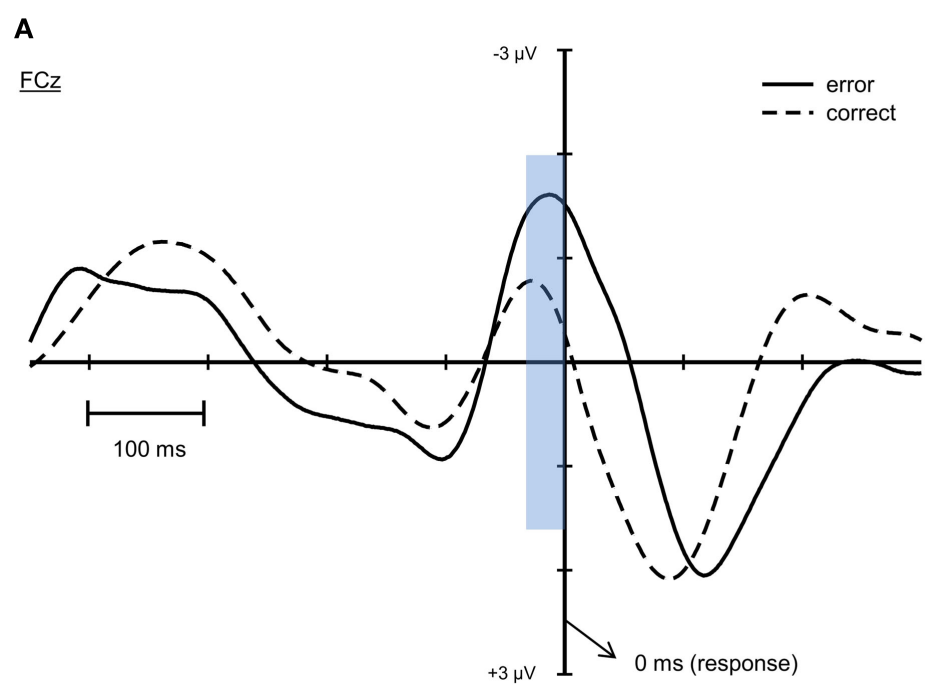

B

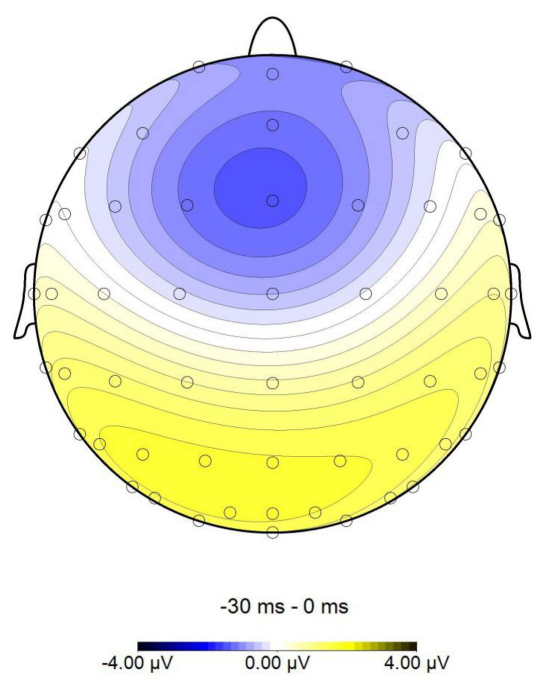

FIGURE 7 | Response-locked ERPs at electrode FCz for erroneous and correct responses (A). Time point 0 denotes the response. A higher negativity was revealed for errors ( $\mathrm{Ne}$ ) compared to correct responses (Nc) that already peaked prior to the given response. (B) shows a typical topography of the Ne with a fronto-central maximum in an interval from -30 to $0 \mathrm{~ms}$. in visual areas was necessary for the attentional selection of relevant information in the presence of a strong and middle motion distractor. This was not required when a weak motion transient was presented and the $\mathrm{N} 1$ asymmetry already indicated attentional selection of the luminance change (Wascher and Beste, 2010a,b). The further processing of relevant information was represented by an increase in posterior activation contralateral to the relevant luminance change in the $\mathrm{N} 2$ time window (N2pc). Therefore, this $\mathrm{N} 2 \mathrm{pc}$ might reflect an attentional bias increasing the signal quality of relevant information by using iterative connections in visual areas. With respect to the behavioral data, the additional processing of relevant information might also have led to heightened RT when strong and middle motion distractors were presented in the LOB condition. These results indicate that change detection errors already occurred during early sensory processing. When the sensory representation of the luminance change in the initial spatial map was too weak to compete against the motion transient, this irrelevant transient captured attention (Desimone and Duncan, 1995; Desimone, 1998; Chelazzi et al., 2001). Thus the sharpening of relevant information in visual areas was disrupted, indicated by the absence of an N2pc (see Figure 4A), and the luminance change could not be selected for further processing.

The question arises why already in range of the visual N1 different activation patterns were observed for erroneous compared to correct change detection. A possible explanation could be a differing state of task focus during the experiment. When participants were focused on the task, perception should be better biased in favor of intended information, while response errors and missed responses should more likely appear under an unfocused state. According to the biased competition account of visual selective attention, top-down mechanisms can bias the activation in visual areas toward relevant upcoming stimuli (Chelazzi et al., 2001; Reynolds and Desimone, 2003). This effect can be described as a sensory gating of information in the visual system and is shown when observers attend to certain features or locations (Johannes et al., 1995; Mangun, 1995). Referred to the current results, an unfocused state might have led to a reduced effect of this top-down bias and thus to a heightened perceptual conflict with irrelevant stimuli compared to correct change detection (cf. Figures 4A,B).

For erroneous change detection, the top-down filtering of relevant information, represented in the $\mathrm{N} 2 \mathrm{pc}$, might also be impaired as a result of a flaw in the high-level system that is responsible for allocating attentional resources for conflict control. The fronto-central N2 component is associated with the detection of conflict on different levels of information processing (Van Veen and Carter, 2002b; Yeung and Cohen, 2006). The neural generator of this component has been localized in the ACC, an area that is related to conflict detection and the triggering of compensatory top-down processes (Botvinick et al., 1999; van Veen and Carter, 2002a). Therefore, a modulation of fronto-central N2 activation was observed in a study using different levels of perceptual conflict in a visual spatial attention task (Kehrer et al., 2009). In the present experiment, activation at fronto-central electrodes was increased for erroneous change detection compared to correct change detection in the LOB condition (see Figures 6A,B). This effect might result from differences in the response-related activation that overlap with the N2. Yet, this interpretation can be precluded, because there was also a higher activation for erroneous compared to correct change detection, when only missed responses were included in the analysis. Additionally, no difference in fronto-central activation was observed between LOB trials including response errors and those including missed responses. This suggests that the fronto-central N2 in the present experiment depicts a conflict on stimulus level that was successfully detected for both error types and was increased compared to correct change detection (cf. Figure 4A). 
In support of this view, the $\mathrm{N} 2$ activation was also modulated by the conflict induced in the change conditions containing a relevant luminance change. The lowest activation was observed in the unilateral LUM condition and activation increased when a motion distractor was presented, above all when it was spatially separated to the target (see Figure 6A). Therefore, the difference wave of the LOB and LUM change condition (see Figure 6B) showed a typical fronto-central scalp distribution of increased activation in the N2 time window for the LOB condition (Kopp et al., 1996; Van Veen and Carter, 2002b). While in the LUM condition only a single luminance change was presented and thus no perceptual conflict was induced, additional top-down control processes were required when the detection of relevant luminance changes was affected by spatially separated distractors.

Results concerning the Ne further resolve the nature of response errors in the current task. Typically, the $\mathrm{Ne}$ is defined as a negative deflection with a fronto-central maximum that peaks in an interval from 50 to $150 \mathrm{~ms}$ after an incorrect response (Falkenstein et al., 1990, 2000; Gehring et al., 1990, 1993). Also the present analyses revealed a negative peak with a fronto-central scalp distribution (see Figure 7B) that was higher in amplitude for error trials compared to correct trials. Yet, a negative peak was already shown prior to the response for both conditions (see Figure 7A). The $\mathrm{Ne}$ is associated with error detection based on a comparison of the representations of the actual response and the required response (Gehring et al., 1993; Falkenstein et al., 2000). When the system first detects that an ongoing event is worse than expected, a resulting phasic decrease in mesencephalic dopaminergic activity leads to a disinhibition of neurons in the ACC and thus elicits the $\mathrm{Ne}$ (Holroyd and Coles, 2002; Beste et al., 2008, 2009). Erroneous change detection in the present task was associated with an insufficient sensory representation of the luminance change at perceptual processing stages. Already prior to response initiation, the detection of this perceptual error might have led to the determination that luminance change detection proceeded worse than expected, leading to a comparatively early Ne-like activation in the response-locked ERP. Accordingly, incorrect responses at the side of the distractor might have been due to guessing when the perceptual processing of relevant information already failed. A response-independent error detection mechanism should lead to the same activation when responses were guessed correctly and might also have caused the slight Ne-like negativity observed prior to correct responses (Falkenstein et al., 2000). Consequently, both the increased negativity for erroneous compared to correct change detection in the stimulus-locked ERP (fronto-central N2) and for response errors compared to correct responses $(\mathrm{Ne})$ might be attributed to a heightened conflict on perceptual level. This interpretation of results could be further investigated by looking at the probability of response errors and correct responses subsequent to an erroneous capture of attention toward the distractor. Assumed

\section{REFERENCES}

Beste, C., Saft, C., Konrad, C., Andrich, J., Habbel, A., Schepers, I., Jansen,

A., Pfleiderer, B., and Falkenstein, M. (2008). Levels of error processing in Huntington's disease: a combined study using event-related

that response errors result from guessing when an error occurred at the stage of perceptual processing, there should be an equal probability of subsequent response errors and correct responses. Further research based on single trial analyses will be required to prove this assumption.

The fact that the mechanism for conflict and error detection remained functional even when target processing was already affected on sensory level, indicates that the relevant luminance change must still have been represented at higher cognitive levels. Yet, recent studies suggest that this representation can be created in the absence of awareness and might be based on the feed-forward sweep of processing (O'Connell et al., 2007; Hughes and Yeung, 2011; van Gaal and Lamme, 2011; van Gaal et al., 2011). However, the report of the luminance change requires the iterative processing of information between higher and lower order areas (Lamme, 2003). The present study shows that this mechanism can be disrupted when the sensory representation of relevant information in visual areas is weak and attention is captured by an irrelevant competing stimulus. An approach for further investigating these processes might be to concentrate on the interaction of higher and lower order areas in attentional selection. Source connectivity analyses with EEG that measure the effective connectivity between brain areas deliver a method for quantifying the directed influence that one neuronal system exerts over another (Friston, 1994; Schoffelen and Gross, 2009). Further research should focus on investigating in what way the effective connectivity between higher executive areas that are associated with top-down control and visual areas is affected when a distracting stimulus disturbs the report of relevant information.

In conclusion, the present study showed that the ability of an irrelevant motion transient to affect the detection of a spatially separated luminance change depended on the salience of this motion transient. By comparing posterior ERPs between trials containing a correct or incorrect response to these stimuli, we showed that change detection required a stronger sensory representation of the luminance change compared to the motion distractor (i.e., attentional selection of the luminance change). If this could not be accomplished by sensory gating mechanisms in the N1 time window, the sharpening of relevant information, represented in an N2pc, was required for change detection. Erroneous change detection occurred when the initial sensory representation of the luminance change was too weak to compete against the motion transient. Consequently, although high-level mechanisms for conflict and error detection remained intact, the further processing of relevant information that might proceed within iterative connections between higher and lower order areas was affected and the signal strength of the luminance change remained insufficient for giving a correct report in the presence of a conflicting motion distractor (Di Lollo et al., 2000; Enns and Di Lollo, 2000; Lamme and Roelfsema, 2000; Fahrenfort et al., 2007).

LTP- and LTD-like exposure-based visual learning. Curr. Biol. 21, 876-882.

Beste, C., Willemssen, R., Saft, C., and Falkenstein, M. (2009). Error processing in normal aging and in basal ganglia disorders. Neuroscience 159, 143-149.

Botvinick, M., Nystrom, L. E., Fissell, K., Carter, C. S., and Cohen, J. D. (1999). Conflict monitoring versus selection-for-action in anterior cingulate cortex. Nature 402, 179-181. 
Botvinick, M. M., Braver, T. S., Barch, D. M., Carter, C. S., and Cohen, J. D. (2001). Conflict monitoring and cognitive control. Psychol. Rev. 108, 624-652.

Carter, C. S., Braver, T. S., Barch, D. M., Botvinick, M. M., Noll, D., and Cohen, J. D. (1998). Anterior cingulate cortex, error detection, and the online monitoring of performance. Science 280, 747-749.

Chelazzi, L., Duncan, J., Miller, E. K., and Desimone, R. (1998). Responses of neurons in inferior temporal cortex during memory-guided visual search. J. Neurophysiol. 80, 2918-2940.

Chelazzi, L., Miller, E. K., Duncan, J., and Desimone, R. (2001). Responses of neurons in macaque area V4 during memory-guided visual search. Cereb. Cortex 11, 761-772.

Crick, F., and Koch, C. (2003). A framework for consciousness. Nat. Neurosci. 6, 119-126.

Desimone, R. (1998). Visual attention mediated by biased competition in extrastriate visual cortex. Philos. Trans. R. Soc. Lond. B Biol. Sci. 353, 1245-1255.

Desimone, R., and Duncan, J. (1995). Neural mechanisms of selective visual attention. Annu. Rev. Neurosci. 18, 193-222.

Di Lollo, V., Enns, J. T., and Rensink, R. A. (2000). Competition for consciousness among visual events: the psychophysics of reentrant visual processes. J. Exp. Psychol. Gen. 129, 481-507.

Duncan, J. (2006). EPS Mid-Career Award 2004: brain mechanisms of attention. Q. J. Exp. Psychol. (Colchester) 59, 2-27.

Eimer, M. (1995). Event-related potential correlates of transient attention shifts to color and location. Biol. Psychol. 41, 167-182.

Eimer, M., and Kiss, M. (2008). Involuntary attentional capture is determined by task set: evidence from event-related brain potentials. J. Cogn. Neurosci. 20, 1423-1433.

Enns, J. T., and Di Lollo, V. (2000). What's new in visual masking? Trends Cogn. Sci. 4, 345-352.

Fahrenfort, J. J., Scholte, H. S., and Lamme, V. A. (2007). Masking disrupts reentrant processing in human visual cortex. J. Cogn. Neurosci. 19, 1488-1497.

Falkenstein, M., Hohnsbein, J., Hoormann, J., and Blanke, L. (1990). "Effects of errors in choice reaction tasks on the ERP under focused and divided attention," in Psychophysiological Brain Research, eds C. H. M. Brunia, A. W. K. Gaillard, and A. Kok. (Tilburg: Tilburg University Press), 192-195.
Falkenstein, M., Hoormann, J., Christ, S., and Hohnsbein, J. (2000). ERP components on reaction errors and their functional significance: a tutorial. Biol. Psychol. 51, 87-107.

Folstein, J. R., and Van Petten, C. (2008). Influence of cognitive control and mismatch on the N2 component of the ERP: a review. Psychophysiology 45, 152-170.

Friston, K. (1994). Functional and effective connectivity in neuroimaging: a synthesis. Hum. Brain Mapp. 2, 56-78.

Gehring, W. J., Coles, M. G., Meyer, D. E., and Donchin, E. (1990). The error-related negativity: an eventrelated brain potential accompanying errors. Psychophysiology 27, 34.

Gehring, W. J., Goss, B., Coles, M. G., Meyer, D. E., and Donchin, E. (1993). A neural system for error detection and compensation. Psychol. Sci. 4, 385-390.

Gehring, W. J., and Knight, R. T. (2000). Prefrontal-cingulate interactions in action monitoring. Nat. Neurosci. 3, 516-520.

Gratton, G., Coles, M. G., and Donchin, E. (1983). A new method for offline removal of ocular artifact. Electroencephalogr. Clin. Neurophysiol. $55,468-484$.

Hickey, C., Di Lollo, V., and Mcdonald, J. J. (2009). Electrophysiological indices of target and distractor processing in visual search. J. Cogn. Neurosci. 21, 760-775.

Hickey, C., Mcdonald, J. J., and Theeuwes, J. (2006). Electrophysiological evidence of the capture of visual attention. J. Cogn. Neurosci. 18, 604-613.

Holroyd, C. B., and Coles, M. G. (2002). The neural basis of human error processing: reinforcement learning, dopamine, and the errorrelated negativity. Psychol. Rev. 109, 679-709.

Hughes, G., and Yeung, N. (2011). Dissociable correlates of response (conflict) and error awareness in errorrelated brain activity. Neuropsychologia 49, 405-415.

Johannes, S., Munte, T. F., Heinze, H. J., and Mangun, G. R. (1995). Luminance and spatial attention effects on early visual processing. Brain Res. Cogn. Brain Res. 2, 189-205.

Kastner, S., and Ungerleider, L. G. (2001). The neural basis of biased competition in human visual cortex. Neuropsychologia 39, 1263-1276.

Kehrer, S., Kraft, A., Irlbacher, K., Koch, S. P., Hagendorf, H., Kathmann, N., and Brandt, S. A. (2009). Electrophysiological evidence for cognitive control during conflict processing in visual spatial attention. Psychol. Res. 73, 751-761.
Kopp, B., Rist, F., and Mattler, U. (1996). N200 in the flanker task as a neurobehavioral tool for investigating executive control. Psychophysiology 33, 282-294.

Lamme, V. A. (2003). Why visual attention and awareness are different. Trends Cogn. Sci. (Regul. Ed.) 7 12-18.

Lamme, V. A., and Roelfsema, P. R. (2000). The distinct modes of vision offered by feedforward and recurrent processing. Trends Neurosci. 23 571-579.

Mangun, G. R. (1995). Neural mechanisms of visual selective attention. Psychophysiology 32, 4-18.

O’Connell, R. G., Dockree, P. M., Bellgrove, M. A., Kelly, S. P., Hester, R., Garavan, H., Robertson, I. H., and Foxe, J. J. (2007). The role of cingulate cortex in the detection of errors with and without awareness: a high-density electrical mapping study. Eur. J. Neurosci. 25, 2571-2579.

O'Regan, J. K., Rensink, R. A., and Clark, J. J. (1999). Change-blindness as a result of 'mudsplashes'. Nature 398, 34.

Pivik, R. T., Broughton, R. J., Coppola, R., Davidson, R. J., Fox, N., and Nuwer, M. R. (1993). Guidelines for the recording and quantitative analysis of electroencephalographic activity in research contexts. Psychophysiology 30 547-558.

Reynolds, J. H., and Desimone, R. (2003). Interacting roles of attention and visual salience in V4. Neuron 37 , 853-863.

Roelfsema, P. R., Lamme, V. A., Spekreijse, H., and Bosch, H. (2002). Figureground segregation in a recurrent network architecture. J. Cogn. Neurosci. 14, 525-537.

Schoffelen, J. M., and Gross, J. (2009). Source connectivity analysis with MEG and EEG. Hum. Brain Mapp. 30, 1857-1865.

Simons, D. J. (2000). Current approaches to change blindness. Vis. cogn. 7, 1-15.

Simons, D. J., and Levin, D. T. (1997). Change blindness. Trends Cogn. Sci. (Regul. Ed.) 1, 261-267.

Simons, D. J., and Rensink, R. A. (2005) Change blindness: past, present, and future. Trends Cogn. Sci. (Regul. Ed.) 9, 16-20.

van Gaal, S., and Lamme, V. A. (2011) Unconscious high-level information processing: implication for neurobiological theories of consciousness. Neuroscientist. doi:10.1177/ 1073858411404078. [Epub ahead of print].

van Gaal, S., Lamme, V. A., Fahrenfort, J. J., and Ridderinkhof,
K. R. (2011). Dissociable brain mechanisms underlying the conscious and unconscious control of behavior. J. Cogn. Neurosci. 23, 91-105.

van Veen, V., and Carter, C. S. (2002a). The anterior cingulate as a conflict monitor: fMRI and ERP studies. Physiol. Behav. 77, 477-482.

Van Veen, V., and Carter, C. S. (2002b). The timing of action-monitoring processes in the anterior cingulate cortex. J. Cogn. Neurosci. 14, 593-602.

van Veen, V., and Carter, C. S. (2005). Separating semantic conflict and response conflict in the Stroop task: a functional MRI study. Neuroimage 27, 497-504.

Vasey, M. W., and Thayer, J. F. (1987). The continuing problem of false positives in repeated measures ANOVA in psychophysiology: a multivariate solution. Psychophysiology 24, 479-486.

Vidal, F., Hasbroucq, T., Grapperon, J., and Bonnet, M. (2000). Is the 'error negativity' specific to errors? Biol. Psychol. 51, 109-128.

Wascher, E., and Beste, C. (2010a). Spatial representations as an emergent feature of perceptual processing. $J$. Psychophysiol. 24, 161-172.

Wascher, E., and Beste, C. (2010b). Tuning perceptual competition. J. Neurophysiol. 103, 1057-1065.

Yeung, N., and Cohen, J. D. (2006). The impact of cognitive deficits on conflict monitoring. Predictable dissociations between the error-related negativity and N2. Psychol. Sci. 17, 164-171.

Conflict of Interest Statement: The authors declare that the research was conducted in the absence of any commercial or financial relationships that could be construed as a potential conflict of interest.

Received: 19 March 2012; accepted: 07 May 2012; published online: 25 May 2012.

Citation: Schneider D, Beste C and Wascher E (2012) Attentional capture by irrelevant transients leads to perceptual errors in a competitive change detection task. Front. Psychology 3:164. doi: 10.3389/fpsyg.2012.00164

This article was submitted to Frontiers in Cognitive Science, a specialty of Frontiers in Psychology.

Copyright (C) 2012 Schneider, Beste and Wascher. This is an open-access article distributed under the terms of the Creative Commons Attribution Non Commercial License, which permits noncommercial use, distribution, and reproduction in other forums, provided the original authors and source are credited. 